\title{
¿Los valores culturales afectan el bienestar humano? Evidencias desde los reportes de investigación ${ }^{*}$
}

Do cultural values affect human well-being? Evidence from research reports

\author{
Andrés GutiÉrRez-CARMOnA ${ }^{\mathrm{a}}$ \\ Universidad Católica del Norte, Chile \\ ORCID: http://orcid.org/0000-0001-7323-0884 \\ Alfonso Urzúa M. \\ Universidad Católica del Norte, Chile \\ ORCID: http://orcid.org/0000-0002-0882-2194
}

a Autor de correspondencia. Correo electrónico: acarmonagutierrez25@gmail.com

Para citar el artículo: Gutiérrez-Carmona, A., \& Urzúa, A. (2019). ¿Los valores culturales afectan el bienestar humano? Evidencias desde los reportes de investigación. Universitas Psychologica, 18(1), 1-12. https://doi.org/10.11144/Javeriana.upsy18-1.vcab

\section{RESUMEN}

El bienestar ha sido estudiado ampliamente en las últimas décadas, y se le ha relacionado con múltiples variables. El presente artículo describe la relación entre el bienestar y los valores culturales, encontrada a través de la investigación empírica. Se realizó una búsqueda bibliográfica en las bases de datos WoS y SCOPUS, considerando como palabras clave valores culturales y bienestar (cultural values \& well-being). Los resultados son diversos y dan cuenta de la influencia del contexto cultural sobre el comportamiento del bienestar en los diferentes grupos humanos, dado que los niveles de bienestar se relacionan de forma diferente con múltiples variables, dependiendo del contexto sociocultural donde se hayan estudiado. Se espera, con esta revisión, poder contribuir a la comprensión de los valores culturales como mediadores en los niveles de bienestar del ser humano.

\section{Palabras clave}

bienestar subjetivo; bienestar psicológico; valores individuales; valores culturales.

\begin{abstract}
Well-being is a concept that has been studied extensively in recent decades and has been associated with multiple variables. This article describes the relationship between well-being and cultural values found through empirical research. A bibliographic search was carried out in the WoS and SCOPUS databases, considering cultural values and well-being as keywords (valores culturales \& bienestar in Spanish). The results are diverse and show the influence of the cultural context on the behavior of well-being in the different human groups, given that the levels of wellbeing are related differently with multiple variables, depending on the socio-cultural context where they have been studied. With this review, we hope to contribute to the understanding of cultural values as mediators in the levels of well-being of the human being.

Keywords

subjective well-being; psychological well-being; individual values; cultural values.
\end{abstract}


A través del tiempo, la búsqueda del bienestar ha sido una importante motivación, generadora de acciones y eventos, incidiendo en la trayectoria sociocultural del colectivo humano. En la actualidad, muchas de las políticas estatales, sociales y económicas orientan sus objetivos hacia la búsqueda del bienestar de la población, en coherencia con el fuerte y amplio desarrollo de la investigación en torno al bienestar humano (Vázquez, Hervás, Rohana, \& Gómez, 2009). Pese a que los seres humanos, desde su origen, han desarrollado conductas que van en la búsqueda tanto del bienestar individual como del colectivo, el significado como las causas y las consecuencias de "estar o sentirse bien" han ido variando en virtud del desarrollo sociohistórico y de la misma evolución humana, y ya, desde hace un par de décadas, se han hecho importantes y concretos esfuerzos por estudiar y desarrollar el bienestar (Muratori \& Bobowik, 2015).

La investigación actual sobre el bienestar se ha derivado de dos perspectivas generales: el enfoque hedónico, que se centra en la felicidad y lo define en función de alcanzar el placer y la evitación del dolor, y el enfoque eudaimónico, el cual se enfila en el desarrollo humano y su autorrealización, y lo determina en la medida en que un ser humano se sienta en plenitud (Ryan \& Deci, 2001). Los estudios en bienestar subjetivo resultan del enfoque hedónico, inspirado en los planteamientos sobre la felicidad de las escuelas de los filósofos hedonistas y epicúreos de la antigua Grecia, quienes la veían a como la acumulación de momentos agradables y la satisfacción de deseos, en ausencia de dolor y aflicciones (Keyes, Ryff, \& Shmotkin, 2002).

Las investigaciones de Diener (Diener, Suh, Lucas, \& Smith, 1999; Diener, Oishi, \& Lucas, 2003) definen bienestar subjetivo (BS) como la manera en que las personas evalúan su satisfacción con la vida, desde sus respuestas afectivas y sus juicios globales. Con base en esto, el BS podría ser comprendido desde dos componentes: uno cognitivo, denominado "satisfacción con la vida" y otro afectivo, llamado "balanza de afectos" (Arita, 2005). El concepto de satisfacción con la vida involucraría una evaluación global y no solo una apreciación positiva de las emociones resultantes del diario vivir, por lo que una vida "excitante" no sería sinónimo de una vida satisfactoria. Sin embargo, el afecto del momento podría influir en la percepción de las experiencias vitales y, por lo mismo, en la evaluación global (Veenhoven, 1994). En tanto, el aspecto afectivo del bienestar subjetivo se establece por la presencia o ausencia de afectos positivos y negativos. Diener y Lucas (1999) señalan que el balance afectivo resulta de una evaluación inmediata y continua de los afectos inducidos por eventos vitales, por lo que existe la posibilidad de estar satisfecho con la propia vida aun cuando se perciban afectos poco gratificantes, en consecuencia, a la hora de evaluar bienestar, resulta importante medir por separado ambos componentes del BS (RodríguezFernández \& Goñi-Grandmontagne, 2011).

Las raíces de la perspectiva eudaimónica vienen de los planteamientos filosóficos de Aristóteles, quien, en su Ética de Nicómaco, estimula al ser humano a vivir de acuerdo con el ideal o criterio de perfección al cual aspira y otorga sentido a la existencia, relacionando la virtud del hombre con la felicidad (Vázquez et al., 2009). Así, podemos decir, que el bienestar eudaimónico es el proceso y consecución de los valores que nos hacen sentir vivos y auténticos y crecer como personas, y no solo la ejecución de las actividades que nos dan placer o nos alejan del dolor (Villar, Triadó, Resamo, \& Osuna, 2003).

Durante el siglo pasado, se establecieron concepciones del bienestar relacionadas con las cualidades de un ser humano maduro (Allport, 1961), su autorrealización (Maslow, 1968) y su funcionamiento pleno (Rogers, 1961). Con base en estos planteamientos, Ryff (1989) propone el concepto de bienestar psicológico, definido como el desarrollo del verdadero potencial de uno mismo y proponiendo un modelo multidimensional sobre la base de seis dimensiones: 1) propósito en la vida (objetivos vitales que permitan dar sentido a la vida), 2) dominio del entorno (habilidad personal para elegir o crear entornos favorables para satisfacer los deseos y necesidades propias), 3) crecimiento personal (empeño por desarrollar 
las potencialidades y seguir creciendo como persona), 4) relaciones positivas con otras personas (mantenimiento de relaciones), 5) autoaceptación (sentirse bien consigo mismo, actitudes positivas hacia uno mismo) y 6) autonomía (capacidad para mantener las convicciones propias con autodeterminación) (Ryff \& Keyes, 1995; Ryff \& Singer, 2006). Sobre la base de estas dimensiones, se puede afirmar que las personas que tienen bienestar psicológico se sienten bien consigo mismas (siendo conscientes de sus propias limitaciones), mantienen relaciones armónicas con los demás, pueden influenciar su medio para satisfacer sus necesidades, cuentan con un propósito de vida y son capaces de desarrollar sus capacidades (Vázquez et al., 2009).

\section{Bienestar y factores relacionados}

Una de las principales dificultades en la investigación del bienestar ha sido establecer con claridad cuáles son las variables que lo determinan y cómo lo hacen, diferenciando las variables predictoras de las que están solo vinculadas (García-Viniegras \& González, 2000). En diferentes estudios se ha reportado cómo variables demográficas (edad, sexo, género, procedencia), de personalidad, las relaciones sociales, las condiciones socioeconómicas y el significado atribuido al vivir bien influyen, en inconstante medida, en el nivel de bienestar de una persona (Vielma \& Alonso, 2010). En el caso de las variables sociodemográficas, diversas investigaciones han demostrado que solo explican entre un 8 y un $20 \%$ de la varianza del bienestar subjetivo (Argyle, 2001). Se ha observado que el bienestar se distribuye en forma de $U$ a lo largo de la vida, comenzando a aumentar durante la juventud y hasta la etapa adulta, para luego empezar a disminuir a partir de la tercera edad (Vera-Villarroel et al., 2012). En el caso del bienestar psicológico, se estima que esta distribución se debe a que las personas más jóvenes y las mayores presentan niveles más bajos en las dimensiones de dominio con el entorno y de autonomía (Eguiluz \& Mera, 2015; Fernández,
Muratori, \& Zubieta, 2013; Ryff, 1989; Zubieta, Muratori, \& Mele, 2012).

Diener, Gohm, Suh y Oishi (2000) establecen que las diferencias en el nivel de bienestar subjetivo entre hombres y mujeres puede deberse, en mayor medida, a la intensidad emocional percibida que a la frecuencia con la que se expresan las emociones (Diener, Gohm, Suh \& Oishi, 2000), siendo los hombres los que demuestran mayor satisfacción con la vida (Atienza, Balaguer, \& García-Merita, 2003). En cuanto al bienestar psicológico, las mujeres puntúan mayores niveles en las dimensiones de relación positiva con los otros y dominio del entorno (Ryff, 1989; Zubieta et al., 2012; Fernández et al., 2013; Muratori, Zubieta, Ubillo, González, \& Bobowik, 2015). Esta diferencia puede ser atribuida al rol tradicional asignado a la mujer que la vincula con el cuidado y la búsqueda del bienestar de los demás (Muratori \& Bobowik, 2015).

Diversas investigaciones han reportado altos niveles de bienestar subjetivo en sociedades con un ingreso económico alto, siendo este último, un predictor moderadamente fuerte de la evaluación de la satisfacción vital, pero uno más débil de los sentimientos positivos y negativos (Diener, Ng, Harter, \& Arora, 2010). Myers y Diener (1995) establecen que la escasez de dinero puede favorecer la tragedia, pero la abundancia no garantiza la felicidad. Diener (1994) establece cuatro posibles explicaciones a la relación bienestar subjetivo e ingresos económicos: 1) en los niveles extremos de pobreza, el ingreso económico tendría un importante efecto en el bienestar de las personas; 2) el status y el poder no aumentan proporcionalmente al ingreso, lo cual podría alterar el bienestar de una persona (según su construcción de estar bien), 3) el efecto del ingreso en el bienestar puede estar determinado por la comparación social que hace cada persona y 4) los ingresos económicos altos, también pueden relacionarse con inconvenientes y/o problemas, los cuales reducen su impacto positivo en la satisfacción vital.

Independiente del ingreso económico, se ha constatado que el contexto en que se vive puede ser un predictor de bienestar; las personas que 
perciben vivir en un entorno positivo, confiable, con relaciones sociales estables presentan un mayor bienestar psicológico (Muratori \& Bobowik, 2015), aun cuando, hayan enfrentado situaciones vitales percibidas como negativas, las cuales también pueden contribuir positivamente al desarrollo personal y al bienestar psicológico, pues las obligan a desarrollar nuevos mecanismos de afrontamiento (Bilbao, Páez, \& MartínezZelaya, 2013).

Los estudios sobre características individuales han mostrado la relación existente entre el bienestar y algunos rasgos de personalidad tales como la extraversión, el neuroticismo y el optimismo o con variables personales como la autoestima (Diener \& Lucas, 1999; VeraVillarroel et al., 2016). Las personas extrovertidas tienen mayor capacidad para construir y mantener amistades, son más emprendedoras, seguras de sí mismas y expresan con mayor facilidad sus emociones, lo cual repercute positivamente en el bienestar (Bisquerra, 2000). Una adecuada autoestima, un acertado nivel de autoeficacia, la capacidad de adaptación, la tolerancia a la frustración y el tener un proyecto de vida, influyen también positivamente en el bienestar (García-Viniegras \& González, 2005). Por su parte, el optimismo ha demostrado ser un importante predictor del bienestar subjetivo (Gustems-Carnicer, Calderón, \& Forn, 2016). Csikszentmihalyi (2005) afirma que las personas con rasgos autotélicos (personalidad con meta autónoma), se perciben a sí mismas de forma positiva, demostrando mayores niveles de bienestar subjetivo. En tanto, las relaciones positivas con los otros influyen positivamente, tanto en el bienestar subjetivo como en el psicológico (Satorres, 2013; Zubieta \& Delfino, 2010; Zubieta et al., 2012).

Se ha comprobado también, que la salud física y el bienestar se relacionan de forma bidireccional. La percepción de salud física influye en la percepción del bienestar, y el sentir bienestar influye en todas las manifestaciones de la esfera humana, incluyendo las acciones que promueven y mantienen la salud. En tanto, altos niveles de bienestar intervienen en la percepción de la salud física (García-Viniegras \& González
Benítez, 2000). Algunas investigaciones han establecido un fuerte nexo entre el bienestar psicológico y la salud física, pues se estima que este tipo de bienestar influye en los mecanismos adaptativos, en las conductas supervivenciales y en las conductas de ajuste a las demandas del medio (Vázquez et al., 2009).

Además de los factores antes descritos, el bienestar está determinado por factores culturales (Schimmack, Radhakrishnan, Oishi, Dzokoto, \& Ahadi, 2002). El contexto sociocultural contribuye al significado de "estar bien", "sentirse bien" o "tener bienestar" (Triandis, 1994; D’Anello, 2006), así como también, al énfasis depositado por la persona en los aspectos privados o relacionales del sí mismo y a la manifestación del individualismo y el colectivismo ( $\mathrm{D}^{\prime}$ Anello, 2006; Suh, Diener, Oishi, \& Triandis, 1998; Triandis, 1994).

Diversos estudios han buscado reconocer el cómo las dimensiones culturales de individualismo y colectivismo afectan en diversos indicadores de bienestar (Urzúa, MirandaCastillo, Caqueo-Urízar, Mascayano, 2013). En culturas individualistas se busca la independencia y la autonomía, y los intereses del grupo son importantes, solo en función de cuánto afectan las propias necesidades del individuo, en cambio, en una cultura colectivista, se busca la interdependencia entre las personas, siendo las necesidades orientadas al bien grupal, las más importantes para el individuo. Otros de los factores que diferencian estos dos tipos de culturas es que las sociedades colectivistas son más herméticas y los individuos pueden llegar a subordinar y suprimir sus emociones y deseos en función del bienestar del grupo (Anguas, 2005; Carr, 2007; Cienfuegos, Saldívar, Díaz, \& Avalos, 2016). Algunos estudios sugieren que las culturas colectivistas, con altos valores de conservación, buscan no alterar la armonía del grupo, por lo cual utilizan mecanismos de afrontamiento que favorecen el autocontrol y restringen las expresiones emocionales (Basabe et al., 2002; Matsumoto, Yoo, \& Nakagawa, 2008). Se ha verificado así, que las culturas individualistas tienen un mayor bienestar subjetivo y las 
colectivistas, un mayor bienestar psicológico (Diener, Suh, Smith, \& Shao, 1995; Muratori \& Bobowik, 2015). Según Vilma y Alonso (2010), la tendencia al colectivismo supone riesgos para el desarrollo y la mantención del bienestar subjetivo, pues enfrenta al individuo a normas y exigencias colectivas que restringen las opciones de disfrute, de satisfacción de sus necesidades psicológicas y de desarrollo de metas personales. En tanto, los estilos de afrontamiento de una cultura colectivista protegen las relaciones interpersonales y promueven la adaptación a los roles contextuales, lo cual hace que estas sociedades tengan mejores niveles de bienestar eudaimónico que de bienestar hedónico (Muratori \& Bobowik, 2015).

\section{Valores culturales e individuales}

El ser humano se mueve motivado por la satisfacción de sus necesidades y por la búsqueda de su bienestar. Estas motivaciones, en general universales, se exploran o se alcanzan a través de particulares caminos, determinados por las construcciones sociales y la cultura de origen (Maslow, 1968). Desde este punto de vista, el proceso de satisfacción involucra una transacción entre el individuo y su entorno micro y macrosocial, influenciada por la trayectoria histórica y el contexto en que se encuentra, siendo múltiples las variables que determinan las oportunidades y el sentido de satisfacción (García-Viniegras \& González Benítez, 2000). Bajo esta mirada, los valores se constituyen en los requisitos regulatorios en la satisfacción de las necesidades biológicas, la coordinación interpersonal y las demandas sociales, en la búsqueda del bienestar individual, del grupo y de la supervivencia (Schwartz \& Bilsky, 1987).

Con base en un sólido desarrollo teórico y empírico, Schwartz (1992) propone conceptualizar los valores como metas amplias, de importancia variable, que subyacen y guían las actitudes y el comportamiento, organizándose en un sistema integro de prioridades. Su propuesta considera una estructura de 10 valores (poder, logro, hedonismo, estimulación, autodirección, universalismo, benevolencia, tradición, conformidad y seguridad), organizados en cuatro bipolaridades (autotrascendencia vs. autopromoción y conservación vs. apertura al cambio) (Schwartz, 2001). Se establece que la relación entre estos valores es dinámica, pues las acciones emprendidas para conseguirlos tienen consecuencias psicológicas, prácticas y sociales, que pueden ser incompatibles con la ejecución de otros tipos de valores. Por ejemplo, perseguir el valor del poder (búsqueda del prestigio social y el control o dominio sobre otras personas) es contrario a la persecución del valor del universalismo (comprensión, aprecio, tolerancia y protección para el bienestar de toda la gente) (Schwartz, 1992, 2006, 2010).

La estructura de 10 valores y su dinámica de relación se han representado en un círculo, donde los valores antagónicos se encuentran en posiciones opuestas al centro del círculo y los valores complementarios, en el lugar contiguo o más cercano. Esta estructura demuestra un continuo motivacional, donde cada valor puede ser inferido de acuerdo a su proximidad o lejanía de otro (Schwartz, 1992). Las dos dimensiones bipolares también se demuestran en este círculo: apertura al cambio (valores de autodirección y estimulación) vs. conservación (valores de conformidad, tradición y seguridad) y autopromoción (valores de logro y poder) vs. autotrascendencia (valores de benevolencia y universalización). La primera polariza la valoración de vivir nuevas experiencias y de la independencia de acciones, pensamientos y sentimientos vs. la seguridad, el orden y la autorrestricción. La segunda bipolaridad manifiesta lo opuesto de perseguir por sobre todo los intereses personales vs. el bienestar de los demás (Schwartz, 1992).

Schwartz $(1992,2006)$ sugiere también que los valores pueden organizarse según sus intereses, sean estos individualistas o colectivistas, estableciendo una estructura bidimensional, donde las personas pueden presentar un perfil valórico individualista (valores de logro, poder, autodirección, estimulación y hedonismo), colectivista (valores conformidad, tradición y benevolencia) o mixto. Los valores de 
seguridad y universalismo, en esta estructura, son considerados como valores mixtos, pues van más allá de la antonimia entre individualismo y colectivismo, demostrando la posibilidad de tener un desarrollo individual que pasa también por el desarrollo colectivo. Por ejemplo, cuando se busca la seguridad, armonía y estabilidad de la sociedad (valor de seguridad) y el bienestar de todos (valor de universalismo), el individuo va en busca tanto de su interés personal como el de la colectividad (Schwartz, 1990).

En este mismo sentido, se ha establecido que los patrones motivacionales individualistas y colectivistas, pueden darse simultáneamente y coexistir en una misma cultura. En un estudio realizado con muestras pertenecientes a un conjunto de países de Europa Occidental (Dinamarca, Grecia, Finlandia, Francia, Holanda, Italia, Portugal, España, Suiza y la antigua Alemania Occidental), se demostró que coexisten ambos tipos de valores (individualistas y colectivistas), pero en distintas combinaciones según el país (Ros \& Schwartz, 1995).

Finalmente, el circulo de los valores se ha podido organizar en una estructura bipolar que contrapone los valores de autoprotección y control de la ansiedad vs. los valores de crecimiento y autoexpansión libre de ansiedad. En los primeros, los valores de poder se relacionan con el control de la ansiedad y las amenazas y el valor de conservación con la aceptación pasiva del statu quo. En cambio, los segundos (valores de crecimiento y autoexpansión) son considerados relativamente libres de ansiedad ( Sortheix \& Schwartz, 2017).

Cuando las culturas son la unidad de análisis, y no los individuos, se habla de valores culturales, los cuales reflejan las diferentes estrategias de regulación de la actividad humana establecida por una sociedad (Schwartz, 1994). Los valores culturales dan cuenta de lo aceptado como correcto y lo deseable dentro de una comunidad, así, las acciones individuales y colectivas emprendidas en el desarrollo de estas motivaciones, son reforzadas por el endogrupo o por la la sociedad misma (Smith \& Schwartz, 1997). Los valores culturales son aprendidos por los individuos durante la socialización primaria, cuando comienzan a ser reconocidos como los intereses principales de la sociedad. Sin embargo, no necesariamente existirá una correspondencia entre estos y los individuales, pues estos últimos también son mediados por la experiencia individual única y personal (Smith \& Schwartz, 1997).

Desde la perspectiva cultural, Schwartz (1994) propone una estructura de tres dimensiones bipolares de valores: conservadurismo vs. autonomía (cercana al aspecto de individualismo y colectivismos) (Ros \& Schwartz, 1995), jerarquía vs. igualitarismo y dominio vs. armonía . Esta estructura sigue la premisa de los valores individuales, en el sentido de que los valores similares se encuentran próximos y los antagónicos lejanos. La primera dimensión (conservadurismo vs. autonomía intelectual y afectiva) polariza las sociedades donde los intereses individuales no se perciben como independientes de los del grupo (valores de conformidad, seguridad y tradición) y aquellas que potencian los intereses individuales, tanto a nivel intelectual como afectivo (valores de autodirección, hedonismo y estimulación).

La segunda dimensión (jerarquía vs. igualitarismo) da cuenta de cómo se distribuyen los recursos, el poder y las oportunidades dentro de en una sociedad. En las culturas jerárquicas, existe un encasillamiento de los individuos y los intereses sociales se abocan en el cumplimiento de las obligaciones y de los roles adscritos, buscando la sanción cuando estos no se cumplen. Las culturas igualitarias conciben a todos los individuos como semejantes, y se valora el respeto y la preocupación por los demás y la cooperación individual en la colectividad.

La tercera dimensión (dominio vs. armonía) se refiere a la actitud de los seres humanos frente al mundo social y natural (sometimiento, adaptación o explotación). En las culturas donde se valora mayormente el dominio, los intereses y acciones irán en el sentido de la dominación y el control del mundo social y natural, explotándolos con el fin de satisfacer las necesidades individuales o grupales. En cambio, en las culturas enfocadas en la armonía, 
se acepta al mundo social y al natural tal como son, buscando la adaptación armónica al entorno (Schwartz, 1994). De la interacción de estos valores se puede determinar también, la aceptación o rechazo de la legitimidad social de perseguir intereses individuales o egoístas vs. la trascendencia de los intereses del yo y del endogrupo, en favor de los demás (dominio y jerarquía vs. igualitarismo y armonía) (Ros \& Schwartz, 1995).

\section{Bienestar y valores}

Diferentes estudios han reportado correlaciones positivas entre bienestar y valores individuales (Bilbao, Techio, \& Páez, 2007; Bobowik, Basabe, Páez, Jiménez, \& Bilbao, 2011; Cohen \& Shamai, 2009; Joshanloo \& Gahedi, 2009; Oishi, Diener, Lucas, \& Suh, 1999; Sagiv \& Schwartz, 2000; Sortheix \& Lönnqvist, 2014; Sortheix \& Schwartz, 2017; Szczesniak, Verhelst, Rondón, \& Straffi, 2013). Los resultados reportados no son concluyentes, pues dependiendo del lugar geográfico donde se realizó el estudio, son distintos los hallazgos. Así, en Alemania e Israel, se encontró que el bienestar subjetivo se relaciona positivamente con la autodirección, y negativamente con los valores de logro y estimulación (Sagiv \& Schwartz, 2000), en tanto en Colombia, se relacionó principalmente con los valores de las dimensiones de autotrascendencia (benevolencia y universalismo) y conservación (tradición, conformidad y seguridad) (Szczesniak, Verhelst, Rondón, \& Straffi, 2013).

En una revisión que involucró cinco estudios (Bilbao et al., 2007), se observó una asociación positiva entre el bienestar subjetivo y los valores conservacionistas y colectivistas y, negativa, con el valor de poder. Los valores de autotrascendencia se relacionaron en forma positiva con la satisfacción vital, observándose sí que estas asociaciones fueron más débiles en los inmigrantes que en los estudiantes de las muestras. Por su parte, los valores de apertura al cambio (estimulación y autodirección) se asociaron fuertemente al bienestar, reportando una mayor vinculación en los inmigrantes que en los autóctonos españoles (más conservacionistas), mientras que los valores individualistas se relacionaron más mesuradamente, en los países de contexto más colectivista, como México y Chile. Los datos anteriores sugieren una moderación del contexto cultural en la relación bienestar y valores.

Otros estudios demostraron que los valores de las dimensiones de autotranscendencia y apertura al cambio se asocian positivamente al bienestar subjetivo (Bobowik et al., 2011; Cohen \& Shamai, 2009; Pascual \& Frías-Navarro, 2012). En el estudio de Sortheix y Schwartz (2017), se encontró que la satisfacción vital presentaba una relación positiva con los valores de autodirección, estimulación, hedonismo y benevolencia y, negativa, a los valores de poder, seguridad, conformidad y tradición. Los valores de universalismo y logro no estuvieron relacionados con la satisfacción vital.

En resumen, se ha corroborado que, en general, los valores de hedonismo, estimulación, autodirección y benevolencia tienden a relacionarse positivamente con el bienestar, en cambio, el valor de poder lo hace negativamente. Por su parte, los valores de tradición, conformidad, seguridad, logro y universalismo no mostraron una tendencia clara. A pesar estas tendencias, el análisis de estos estudios demuestra inconsistencia en estas relaciones. En un intento por aclararlas, Sortheix y Lönnqvist (2014) sugirieron que el Índice de Desarrollo Humano de un país, se constituye como moderador de la relación entre satisfacción vital (componente del bienestar subjetivo) y los valores individuales. Según los investigadores, la asociación entre satisfacción con la vida y valores individuales depende de qué tan bien estos últimos se alinean con el contexto socioeconómico. De acuerdo con lo anterior, en los países con un alto puntaje del índice (altos ingresos económico, acceso igualitario a la educación y alta esperanza de vida), los valores centrados en la sociedad (autotrascendencia y conservación) promoverían la satisfacción con la vida y los valores de autopromoción la debilitarían; esto se explica con 
base en que, en dichas sociedades, la inversión en relaciones sociales cooperativas favorece el bienestar. En contraste con lo anterior, en los países con un bajo índice, los valores centrados en la persona (autopromoción y apertura al cambio) favorecerían la satisfacción con la vida y los valores centrados en el universalismo, la disminuirían. Lo anterior reside en que un contexto de subdesarrollo socioeconómico lleva a que el individuo movilice sus valores de apertura al cambio y autopromoción, para poder adaptarse con éxito a ese entorno (Sortheix \& Lönnqvist, 2014).

Siguiendo esta línea, el estudio de Sortheix y Schwartz (2017) evaluó el papel moderador del índice de desarrollo humano y el igualitarismo cultural en la relación bienestar subjetivo (satisfacción con la vida) y valores. Se estableció que tanto el índice (factores estructurales) como el igualitarismo cultural (referido a valores culturales normativos) proporcionan un contexto favorable para la vida de las personas. Los resultados demostraron que el igualitarismo cultural modera todas las asociaciones entre valores individuales y satisfacción vital. Así, se pudo observar que, en los países menos igualitarios, los valores de autodirección, estimulación y hedonismo se relacionan en forma más positiva con la satisfacción vital y los valores de poder y logro, menos negativamente. Por su parte, los valores de seguridad, conformidad y tradición se asocian más negativamente con satisfacción vital, y los valores de universalismo y benevolencia en forma menos positiva. En sociedades con menor igualitarismo social, los valores de apertura al cambio son útiles para compensar la falta de estabilidad y apoyo social, propias de este tipo de sociedades, mientras que los valores de poder y logro permiten enfrentarse de mejor manera a sociedades más jerárquicas (Sortheix \& Schwartz, 2017). En este estudio, se demostró que el igualitarismo cultural, más que el índice de desarrollo humano, puede ser considerado como un moderador entre los valores individuales y el bienestar subjetivo.

En el caso del bienestar psicológico, se ha comprobado que sus niveles se relacionan positivamente con los valores de hedonismo, estimulación, autodirección, universalismo y el de benevolencia (Bilbao, 2008; Bilbao et al., 2007; Zubieta et al., 2012), y que existe una asociación negativa entre la dimensión del bienestar psicológico de autonomía y los valores de tradición y seguridad (Bilbao, 2008). Así, también, se ha encontrado que en personas adultas mayores, la dimensión de apertura el cambio promueve las emociones positivas y protege de las emociones negativas (Burr, Santo, \& Pushkar, 2011), de lo cual se puede concluir que, al igual que en el bienestar subjetivo, los valores de las dimensiones de apertura al cambio y autotrascendencia se relacionan positivamente con el bienestar psicológico y que los valores de la dimensión de conservación lo hacen en forma negativa (Bilbao, 2008; Bilbao et al., 2007; Zubieta et al., 2012).

\section{Referencias}

Allport, G. W. (1961). Pattern and growth in personality. Nueva York: Holt, Rinehart, \& Winston.

Anguas Plata, A. M. (2005). Bienestar subjetivo en México: un enfoque etnopsicológico. En E. L. Garduño, A. B. Salinas \& H. M. Rojas (Coords.), Calidad de vida y bienestar subjetivo en México (pp. 167-196). México: Plaza y Valdés.

Arita, B. (2005). Satisfacción por la vida y teoría homeostática del bienestar. Psicología y Salud, 15(1), 121-126. Recuperado de htt p://www.redalyc.org/pdf/291/29115113.pdf

Argyle, M. (2001). The psychology of happiness (2.a ed.). Nueva York: Routledge.

Atienza, F. L., Balaguer, I., \& García-Merita, M. (2003). Satisfaction with Life Scale: Analysis of factorial invariance across sexes. Personality and Individual Differences, 35, 1255-1260. https://doi.org/10.1016/S01918869(02)00332-X

Basabe, N., Páez, D., Valencia, J., González, J. L., Rime, B., \& Diener, E. (2002). Cultural dimensions, socioeconomic development, climate and emotional hedonic level. 
Cognition y Emotion, 16(1), 103-125. https: //doi.org/10.1080/02699930143000158

Bilbao Ramírez, M. (2008). Creencias sociales y bienestar: valores, creencias básicas, impacto de los hechos vitales y crecimiento psicológico (Tesis doctoral inédita). Universidad del País Vasco, Lejona, España.

Bilbao, M. A., Techio, E. M., \& Páez, D. (2007). Felicidad, cultura y valores personales: estado de la cuestión y síntesis meta-analítica. Revista de Psicología, 15(2), 233-276. Recuperado de http://pepsic.bvsa lud.org/pdf/rp/v25n2/v25n2a05.pdf

Bilbao, M. A., Páez, D., \& Martínez-Zelaya, G. (2013). Cambio en creencias básicas y crecimiento post estrés: un estudio transversal sobre el fuerte impacto de los hechos positivos sobre el bienestar. Terapia Psicológica, 31 (1), 127-139. https://doi.org/ 10.4067/S0718-48082013000100012

Bisquerra, R. (2000). Educación emocional y bienestar. Barcelona: Ciss-Praxis.

Bobowik, M., Basabe, N., Páez, D., Jiménez, A., \& Bilbao, M. A. (2011). Personal values and well-being among Europeans, Spanish natives and immigrants to Spain: Does the culture matter? Journal of Happiness Studies, 12(3), 401-419. https://doi.org/10.1007/s10 902-010-9202-1

Burr, A., Santo, J. B., \& Pushkar, D. (2011). Affective well-being in retirement: The influence of values, money, and health across three years. Journal of Happiness Studies, 12, 17-40. https://doi.org/10.1007/s 10902-009-9173-2

Carr, A. (2007). Psicología positiva. La ciencia de la felicidad. Barcelona: Paidós.

Cienfuegos-Martínez, I., Saldívar-Garduño, A., Díaz-Loving, R., Avalos-Montoya, A. D. (2016). Iindividualismo y colectivismo: caracterización y diferencias entre dos localidades mexicanas. Acta de Investigación Psicológica, 6(3), 2534-2543. https://doi.org /10.1016/j.aipprr.2016.08.003

Cohen, A., \& Shamai, O. (2009). The relationship between individual values, psychological well-being, and organizational commitment among Israeli police officers. International Journal of Police Strategies $\mathbb{E}$ Management, 33, 30-51. https:/ /doi.org/10.1108/13639511011020584

Csikszentmihalyi, M. (2005). Fluir (Flow). Una psicología de la felicidad (11.a ed.). Barcelona: Kairós.

D'Anello, S. (2006). Efecto de las normas y emociones en los juicios sobre satisfacción con la vida en una muestra venezolana. Revista Interamericana de Psicología, 40(3), 371 - 376. Recuperado de http://www.redal yc.org/pdf/284/28440311.pdf

Diener, E. (1994). El bienestar subjetivo. Intervención Psicosocial, 3, 67-113. Recuperado de https://journals.copmadrid. org/pi/archivos/1994/vol2/arti5.htm

Diener, E., Gohm, C. L., Suh, E., \& Oishi, S. (2000). Similarity of the relations between marital status and subjective well-being across cultures. Journal of Cross-Cultural Psychology, 31(4), 419-436. https://doi.org/ 10.1177/0022022100031004001

Diener, E., \& Lucas R. E. (1999). Personality and subjective well-being. En D. Kahneman, E. Diener \& N. Schwarz (Eds.), Wellbeing: The foundations of hedonic psychology (pp. 213-229). Nueva York: Russell Sage Foundation.

Diener, E., Ng, W., Harter, J., \& Arora, R. (2010). Wealth and happiness across the world: Material prosperity predicts life evaluation, whereas psychosocial prosperity predicts positive feeling. Journal of Personality and Social Psychology, 99, 52-61. https://doi.org/ 10.1037/a0018066

Diener, Ed., Oishi, S., \& Lucas, R. (2003). Personality, culture, and well-being: Emotional and cognitive evaluations of life. Annual Review of Psychology, 54, 403. 425. https://doi.org/10.1146/annurev.psych .54 .101601 .145056

Diener, E., Suh, E., Lucas, R., \& Smith, H. (1999). Subjetive well-being: Three decades of progressener. Psychological Bulletin, 125(2), 276-302. Recuperado de https://media.rickhanson.net/Papers/Su bjectiveWell-BeingDiener.pdf 
Diener, E., Suh, E., Smith, H., \& Shao, L. (1995). National differences in subjective wellbeing: why do they occur? Social Indicator Research, 34, 7-32. https://doi.org/10.1007/ BF01078966

Eguiluz L. L., \& Mera, J. (2015). Medición del riesgo suicida, bienestar psicológico y estructura familiar en estudiantes de la salud. Enseñanza e Investigación en Psicología, 20(1), 96-101. Recuperado de http://www. redalyc.org/articulo.oa?id $=29242798014$

Fernández, O., Muratori, M., \& Zubieta, E. (2013). Bienestar eudaemónico y soledad emocional y social. Boletín de Psicología, 108, 7-23. Recuperado de http://www.uv.es/seoa ne/boletin/previos/N108-1.pdf

García-Viniegras, C. V., \& González Benítez, I. (2000). La categoría del bienestar psicológico: su relación con otras categorías sociales. Revista Cubana de Medicina Integral, 16(6), 586-592. Recuperado de htt p://scielo.sld.cu/scielo.php?script $=$ sci_artt ext\&pid $=$ S0864-21252000000600010

Gustems-Carnicer, J. C., Calderón, M., \& Forn, M. (2016). Psychometric properties of the Life Orientation Test (LOT-R) and its relationship with psychological well-being and academic progress in college students. Revista Latinoamericana de Psicología. https:/ /doi.org/10.1016/j.rlp.2016.05.001

Joshanloo, M., \& Ghaedi, G. (2009). Value priorities as predictors of hedonic and eudaimonic aspects of well-being. Personality and Individual Differences, 47, 294-298. https://doi.org/10.1016/j.paid.200 9.03.016

Keyes, C. L. M., Ryff, C. D., \& Shmotkin, D. (2002). Optimizing well-being: The empirical encounter of two traditions. Journal of Personality and Social Psychology, 82 (6), 1007-1022. https://doi.org/10.1037// 0022-3514.82.6.1007

Maslow, A. H. (1968). Toward a psychology of being (3.a ed., Trad., R. Lowry). Nueva York: Van Nostrand.

Matsumoto, D., Yoo, S. H., \& Nakagawa, S. (2008). Culture, emotion regulation, and adjustment. Journal of Personality and Social
Psychology, 94(6), 925-937. https://doi.org/ 10.1037/0022-3514.94.6.925

Muratori, M., Zubieta, E., Ubillo, S., González, J., \& Bobowik, M. (2015). Felicidad y bienestar psicológico: estudio comparativo entre Argentina y España. Psykhe, 24(2), 1-18. https://doi.org/10.7764/psykhe.24.2.9 00

Myers, D., \& Diener, E. (1995). Who is happy? Psychological Science, 6(1), 10-19. https://do i.org/10.1111/j.1467-9280.1995.tb00298.x

Oishi, S., Diener, E. F., Lucas, R. E., \& Suh, E. M. (1999). Cross-cultural variations in predictors of life satisfaction: Perspectives from needs and values. Personality and Social Psychology Bulletin, 25 (8), 980-990. http://d x.doi.org/10.1177/01461672992511006

Pascual, M., \& Frías-Navarro, D. (2012). Valores personales asociados al bienestar subjetivo en personas mayores: evidencias a partir de la encuesta social europea. Metodología de Encuestas, 14, 81-101. Recuperado de http://casus.usal.es/pkp/index.php/MdE/ article/viewFile/1027/972

Rodríguez-Fernández, A., \& GoñiGrandmontagne, A. (2011). La estructura tridimensional del bienestar subjetivo. Anales de Psicología, 27(2), 327-332. Recuperado de https://dialnet.unirioja.es/se rvlet/articulo? codigo $=3628450$

Rogers, C. R. (1961). On becoming a person. Boston: Houghton Mifflin.

Ros, M., \& Schwartz, S. H. (1995). Jerarquía de valores en países de la Europa Occidental: una comparación transcultural. Reis: Revista Española de Investigaciones Sociológicas, 69, 69-88. Recuperado de http://www.reis.cis.e s/REIS/PDF/REIS_069_05.pdf

Ryan, R., \& Deci, E. (2001). On happiness and human potentials: A review of research on hedonic and eudaimonic wellbeing. Annual Review of Psychology, 52, 141-166. https://doi.org/10.1146/annurev. psych.52.1.141

Ryff, C. D. (1989). Happiness is everythings, or is it? Exploration on the meaning of psychological wellbeing. Journal of Personality and Social Psychology, 57(6), 
1069-1081. Recuperado de http://aging.wis c.edu/pdfs/379.pdf

Ryff, C. D., \& Keyes, C. (1995). The structure of psychological well-being revisited. Journal of Personality and Social Psychology, 69(4), 719-727. Recuperado de http://midus.wisc. edu/findings/pdfs/830.pdf

Ryff, C. D., \& Singer, B. H. (2006). Best news yet on the six-factor model of well-being. Social Science Research, 35(4), 1103-1119. https:// doi.org/10.1016/j.ssresearch.2006.01.002

Sagiv, L., \& Schwartz, S. H. (2000). Value priorities and subjective well-being: Direct relations and congruity effects. European Journal of Social Psychology, $30(2), \quad 177-198$. https://doi.org/10.1002/ (SICI) 1099-0992 (200003/04)30:2<177::AID, EJSP982>3.0.CO;2-Z

Satorres, E. (2013). Bienestar psicológico en la vejez y su relación con la capacidad funcional y la satisfacción vital (Tesis de doctorado inédita). Universidad de Valencia, España. Recuperado de http://roderic.uv.es/bitstream/handle/10 550/26298/envejecimiento\%20y\%20bienes tar.pdf? sequence $=1$

Schimmack, U., Radhakrishnan, P., Oishi, S., Dzokoto, V., \& Ahadi, S. (2002). Culture, personality, and subjective wellbeing. Integrating process models of life satisfaction. Journal of Personality and Social Psychology, 82 (4), 582-593. https://doi.org/ 10.1037/0022-3514.82.4.582

Schwartz, S. H. (1990). IndividualismCollectivism: Critique and proposed refinements. Journal of Cross-Cultural Psychology, 21(2), 139-157. https://doi.org/ 10.1177/0022022190212001

Schwartz, S. H. (1992). Universals in the content and structure of values: Theory and empirical tests in 20 countries. Advances in Experimental Social Psychology, 25, 1-65. https://doi.org/10.1016/S0065-26 01(08)60281-6

Schwartz, S. H. (1994). Are there universals aspects in the structure and contents of human values? Journal of Social Issues,
50(4), 19-45. https://doi.org/10.1111/j.154 0-4560.1994.tb01196.x

Schwartz, S. (2001). ¿Existen aspectos universales en la estructura y contenido de los valores humanos? En M. Ros \& V. Gouveia (Eds.), Psicología social de los valores humanos (pp. 53-76). Madrid: Biblioteca Nueva.

Schwartz, S. H. (2006). Les valeurs de base de la personne: Théorie, mesures et applications [Valores básicos de la persona: teoría, medidas y aplicaciones]. Revue Française de Sociologie, 47, 929-968. Recuperado de http://www.cairn.info/revue-francaise-d e-sociologie-2006-4-page-929.htm

Schwartz, S. H. (2010). Basic values: How they motivate and inhibit prosocial behavior. En M. Mikulincer \& P. R. Shaver (Eds.), Prosocial motives, emotions, and behavior: The better angels of our nature (pp. 221-241). Washington, DC: American Psychological Association.

Schwartz, S. H., \& Bilsky, W. (1987). Toward a universal psychological structure of human values. Journal of Personality and Social Psychology, 53(3), 550-562. https://doi.org/ 10.1037/12061-012

Smith, P. B., \& Schwartz, S. H. (1997). Values. En J. W. Berry, M. H. Segall \& C. Kagitcibasi (Eds.), Handbook of cross-cultural psychology (2.a ed., Vol. 3, pp. 77-118). Boston, MA: Allyn \& Bacon.

Sortheix, F. M., \& Lönnqvist, J. E. (2014). Personal value priorities and life satisfaction in Europe: The moderating role of socioeconomic development. Journal of Cross-Cultural Psychology, 45(2), 282-299. https://doi.org/10.1177/00220221 13504621

Sortheix, F. M., \& Schwartz, S. H. (2017). Values that underlie and undermine wellbeing: Variability across countries. European Journal of Personality, 31 (2), 187-201. https ://doi.org/10.1002/per.2096

Suh, E., Diener, E., Oishi, S., \& Triandis, H. (1998). The shifting basis of life satisfaction judgments across cultures: Emotions versus Norms. Journal of Personality and Social 
Psychology, 74(2), 482-493. https://doi.org/ 10.1037/0022-3514.74.2.482

Szczesniak, M., Verhelst, T., Rondón, G. P., \& Straffi, E. (2013). iTener o ser? La estructura de los valores y bienestar subjetivo de los padres colombianos. Informes Psicológicos, 13(1), 53-64. Recuperado de https://revistas.upb.edu.co/index.php/in formespsicologicos/article/view/2343/2087

Triandis, H. C. (1994). Culture and social behavior. Nueva York: McGraw-Hill.

Urzúa, A., Miranda-Castillo, C., CaqueoUrízar, A., Mascayano, F. (2013). Do cultural values affect quality of life evaluation? Social Indicators Research, 112(6), 1295-1313. https://doi.org/10.1007 /s11205-012-0203-9

Vázquez, C., Hervás, G., Rohana, J. J., \& Gómez, D. (2009). Bienestar psicológico y salud: aportaciones desde la psicología positiva. Anuario de Psicología Clínica y de la Salud, 5, 15-28. Recuperado de http://institucionale s.us.es/apcs/doc/APCS_5_esp_15-28.pdf

Veenhoven, R. (1994). El estudio de la satisfacción con la vida. Intervención Psicosocial, 3(9), 87-116. Recuperado de ht tp://hdl.handle.net/1765/16195

Vera-Villarroel, P., Celis-Atenas, K., Pavez, P., Lillo, S., Bello, F., Díaz, N., \& López, W. (2012). Dinero, edad y felicidad: asociación del bienestar subjetivo y variables sociodemográficas. Revista Latinoamericana de Psicología, 44, 155-164. https://doi.org/1 0.4067/S0718-48082012000200008

Vera-Villarroel, P., Celis, K., Urzúa, A., Silva, J., Contreras, D., \& Lillo, S. (2016). Los afectos como mediadores de la relación optimismo y bienestar. Revista Argentina de Clínica Psicológica, 25(2), 195-202. Recuperado de http://www.redalyc.org/pdf/ 2819/281946990010.pdf

Vielma, J., \& Alonso, L. (2010). El bienestar psicológico subjetivo en estudiantes universitarios: La evaluación sistemática del flujo en la vida cotidiana (Tesis doctoral inédita). Universidad de los Andes, Mérida, Venezuela.
Villar, F., Triadó, C., Resamo, S., \& Osuna, M. (2003). Bienestar, adaptación y envejecimiento: cuando la estabilidad significa cambio. Revista Multidisciplinar de Gerontología, 13(3), 152-162. Recuperado de http://www.ub.edu/dppsed/fvillar/princi $\mathrm{pal} / \mathrm{pdf} / 2003$ revista_multidisciplinar_ada ptacion.pdf

Zubieta, E. M., \& Delfino, G. I. (2010). Satisfacción con la vida, bienestar psicológico y bienestar social en estudiantes universitarios de Buenos Aires. Anuario de Investigaciones, 17, 277-283. Recuperado de http://www.redalyc.org/pdf/3691/36913 9946018.pdf

Zubieta, E., Muratori, M., \& Fernández, O. (2012). Bienestar subjetivo y psicosocial: explorando diferencias de género. Salud Eु Sociedad, 3(1), 66-76. Recuperado de http://pepsic.bvsalud.org/pdf/salsoc/v3n 1/a05.pdf

Zubieta, E. M., Muratori, M., \& Mele, S. (2012). Bienestar, clima emocional, percepción de problemas sociales y confianza. Anuario de Investigaciones, 19(1), 97-106. Recuperado de http://www.redalyc.org/pdf/3691/36913 9948009.pdf

\section{Notas}

* Artículo de revisión 